A côté, dans la même impureté, on trouve des vaisseaux, des poils végétaux, des enveloppes cellulaires, variables dans leur as pect et suivant leur provenance, mais toujours reconnaissables à leurs réactions microchimiques.

Los vaisseaux des tissus végétaux qui constituent les impuretés que nous examinons en ce moment sont annelés; on en trouve de spiralés, entiers ou brisés. A côté, il y a des poils d'aspect vitreux, des cellules polyédriques, fusiformes ou en palissades, etc... (Voir fig. 6, 8,9 et 10 )

Ces diverses particules, dont le microscope permet de reconnaître l'origine végétale à travers les transformations qu'elles ont subies dans la traversée du tube digestif, présentent des réactions microchimiques. La plus intéressante est celle que donne la cellulose. Entre lame et lamelle, une goutte d'acide sulfurique simplifie la cellulose, la ramène partiellement à l'état d'amidon, ou, ce qui peut-être est plus exact, en attaquant les parois cellulaires, met en liberté l'amidon que renfermaient les cellules. Si l'on ajoute une goutte de solution iodée, on note alors des taches et des traînées plus foncées.

(A suivre.)

\title{
LA MÉTHOdE SYNTHÉTIQUE DANS L'ÉTUDE DU LAIT. LE LAIT AU POINT DE VUE COLLOIDAL. RECHERCHES SUR LE MÉCANISIME DE L'ACTION DE LA PRÉSURE
}

\author{
par Ch. PORCHER,
}

Docteur ès sciences physiques

(Suite)

Voyons d'abord les expériences qui nous permettent d'apprécier dans le complexe la sensibilité, vis-à-vis de la chaleur, du caséinate calcique et du phosphate calcique; puis, à la lumièrefdes enseignements que nous tirerons de la mézanique chimique des phosphates de calcium, nous nous emploierons à donner une interprétation sur une base ehimique, - et non plus cette fois colloïdale, - de l'inaptitude du com. plexe, chauffé à haute température, à coaguler par la présure.

Nous disposons de deux moyens pour montrer que, dans l'action de la chaleur sur le complexe, ce sont les phosphates calciques qui sont particulièrement atteints :

1 1 $\mid$ Chauffage d'un caséinate neutre ou voisin de la neutralité, puis chargé, à la température ordinaire, en!p hosp hates calciques. - Des portions égales d'un caséinate $(p H=7,03)$ sont chauffées 30 minutes à $40^{\circ}$, à $65^{\circ}$, à $80^{\circ}$ et à $100^{\circ}$. Le $p H$ n'est pas altéré par ce chauffage. A ces caséinates, 
on ajoute des mêmes quantités d'eau de chaux que l'on neutralise ensuite par de l'acide phosphorique pour obtenir le même $p$ H (ici : 6,78 ) tout en réalisant bien entendu, la charge normale en phosphates calciques. Les temps de prise des complexes ainsi obtenus par la présure à $40^{\circ}$ sont sensiblement les mêmes.

TABLEAU LXXXIX.

Complexes

Temps de prise

Témoin $3 \mathrm{~min} .50 \mathrm{sec}$.

Caséinate chauffé 30 minutes à $40^{\circ}$...... . 3 » 50 》

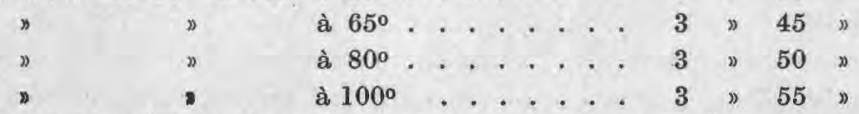

Les caillés dans les quatre cas ont la même consistance : ils sont fermes.

On peut conclure de cette expérience que les caséinates neutres ou très voisins de la neutralité, même chauffés à $100^{\circ}$ pendant 30 minutes, ne sont pas altérés, et si on les additionne de phosphates de calcium à la température ordinaire, on aboutit nécessairement à des complexes identiques au témoin. Avec les caséinates acides $(p H<7)$, on obtient $a$ fortiori les mêmes résultats.

$2^{\circ}$ Action de la chaleur sur le complexe acide $(p \mathrm{H}<7)$ chauffé, puis rechargé en phosphates de calcium neufs. - Dans une première expérience, un complexe dont le $p$ H est de 6,69 est divisé en portions égales qui sont chauffées 30 minutes à $40^{\circ}$, à $65^{\circ}$, à $80^{\circ}$ et à $100^{\circ}$.

Les temps de prise et l'aspect des caillés sont les suivants :

TABLEAU XC.

Témoin

$\begin{array}{ccc}\text { TABLEAU XC. } & \text { Temps de } \\ \text { Complexes } & p \text { prise } & \begin{array}{c}\text { Aspect des } \\ \text { caillés }\end{array}\end{array}$

Chaufté 30 minutes à $40^{\circ} \ldots . .6 .69$

$4 \mathrm{~m}, 30 \mathrm{~s}$. Ferme.

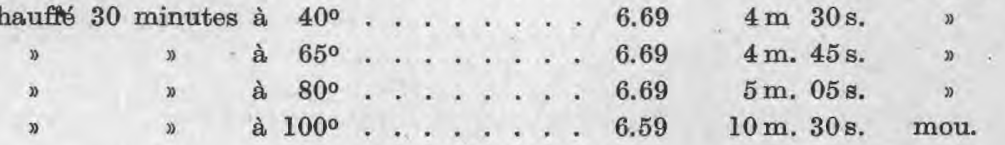

Le $p H$ n'a subi qu'une petite diminution par le chauffage à $100^{\circ}$.

Une autre expérience nous a donné des résultats analogues, mais encore plus marqués, car nous avons porté le complexe jusqu'à $125^{\circ}$.

\section{TABLEAU XCI.}

Complexes

Témoin ........

$7 \mathrm{~m} .30 \mathrm{~s}$.

\section{Aspect des}

caillés

Ferme.

Moins ferme que le précédent.

6.67 Ne coagule pas.

» à $125^{\circ} \ldots \ldots .6 .67$

L'altération du complexe déjà nette à $100^{\circ}$, est telle à $125^{\circ}$ que le complexe ne coagule plus. 
Si nous rechargeons les complexes ainsi chauffés, avec des phosphates de calcium neufs, par addition d'eau de chaux, puis neutralisation subséquente avec $\mathrm{PO}^{4} \mathrm{H}^{3}$, en nous efforçant d'arriverau même $p$ Heten assurant une charge sensiblement de même importance que la première, nous allons voir réapparaître la possibilité de coaguler par la présure.

\section{TABLEAU XCII.}

\begin{tabular}{|c|c|c|c|}
\hline & Complexes & $p$ H & $\begin{array}{c}\text { Temps de } \\
\text { prise }\end{array}$ \\
\hline Témoin & $\ldots . . .$. & 6.87 & $2 \mathrm{~m}$ \\
\hline Chauffé & à $100^{\circ} \ldots \ldots$. & 6.73 & $4 \mathrm{~m}$. \\
\hline$"$ & à $125^{\circ} \ldots . .$. & 6.73 & \\
\hline 》 & à $100^{\circ}$ rechargé. . . & 6.78 & $2 \mathrm{~m} .10 \mathrm{~s}$ \\
\hline$n$ & à $125^{\circ}$ rechargé. . & 6.78 & $6 \mathrm{~m} .15 \mathrm{~s}$ \\
\hline
\end{tabular}
Aspect des caillés
Ferme.
Assez ferme.
$\mathrm{Ne}$ coagule pas.
Ferme ; analogue au caillé témoin.
Compact, mais un peu moins ferme que celui du té- moin.

Incontestablement, les séries d'expériences qui précèdent nous montrent que dans l'action de la chaleur sur le complexe, ce sont les phosphates qui sont atteints.

Voyons maintenant comment nous pourrions lèser les micelles du caséinate et apprécier lainsi le trouble apporté par l'altération de ces derniers dans la façon de se comporter du complexe vis-à-vis de la présure (282).

Deux moyens s'offrent à nous :

10 Action de la chaleur sur des caséinates calciques alcalins, puis' charge de ceux-ci, à la température ordinaire, en phosphates de calcium. - Quand un caséinate est [nettement alcalin $(p H>9)$, l'action de la chaleur l'altère d'autant plus que la réaction est plus franchement alcaline et que le chauffage a duré plus longtemps; il se détache de l'acide phosphorique de la molécule protéique.

Des portions égales d'un caséinate à $1100(p H=9.76)$ sont chauffées 30 minutes à $40^{\circ}, 65^{\circ}, 80^{\circ}$ et $100^{\circ}$.

Mais auparavant, voyons l'allure du $p H$ du caséinate sous l'action de la chaleur:

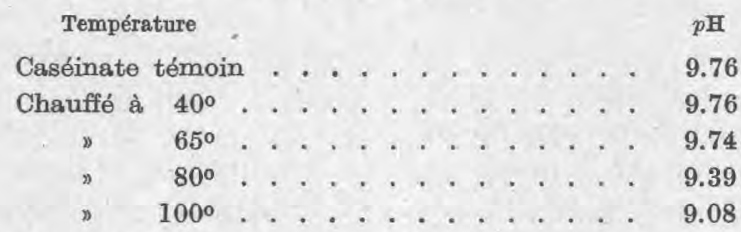

Le $p \mathrm{H}$ a viré vers l'acidité.

(282) Ch. Porcher. - L'altération des micelles du caséinate dans le complexe caséinate le chaux + phosphate de chaux. Ses conséquences dans l'action de la présure sur ce complexe. C. R. Ac. Sc. 1926, $182,1420$. 
Retenons que jusqu'd $65^{\circ}$, il ne semble pas qu'il y ait une altération. Nous savons que $65^{\circ}$ est une température critique que nous retrouverons dans toutes les réactions auxquelles le complexe ou le lait peuvent étre soumis.

Nous n'avons pas à être surpris de cette marche vers l'acidité qui traduit une altération du caséinate, puisque nous savons qu'en présence d'une alcalinisation forte, la caséine est en quelque sorte saponifiée et que l'acide phosphorique qui lui était auparavant lié, s'en sépare. En effet, par chauffage à $100^{\circ}$, il se dépose sur les parois du tube des grains menus dans lesquels on décèle la présence de l'acide phosphorique sans liaison protéique. Il est bien évident que si l'on part des caséinates ci-dessus qui viennent d'être chauffés, pour obtenir des complexes, nous devons aboutir à des ensembles qui ne réagiront pas de la même façon vis-à-vis de la présure. En effet, nous les avons chargés en phosphates de calcium à la température ordinaire par addition d'acide phosphorique et ensuite soumis à l'action de la présure. Voici les résultats obtenus :

TABLEAU XCIII.

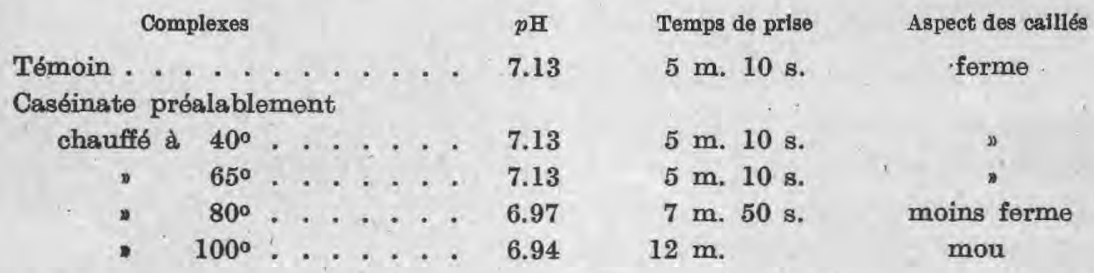

Les différences dans les temps d'emprésurage et surtout dans l'aspect des caillés, nous permettent de conclure qu'il y a une altération marquée de la caséine par le chauffage à partir de $65^{\circ}$.

Il est certain que si l'on avait prolongé l'action des températures nocives comme $80^{\circ}$ et surtout $100^{\circ}$, nous aurions abouti à des systèmes qui n'auraient plus coagulé du tout par la présure.

2० ACTION DU FORMOL A CHAUD SUR LES CASÉINATES. - Il nOus est encore possible d'apprécier l'altération du caséinate dans le complexo en ayant recours au formol.

Nous avons $\mathrm{vu}$ plus haut que lorsqu'on ajoute du formol au lait, il faut des doses vraiment considérables de ce composé pour que l'emprésurage labique en soit impressionné. Il est vrai que le lait est un milieu très complexe et que le formol fait porter son action tout aussi bien sur les protéines qui entourent la caséine : globuline et albumine, que sur la caséine elle-même.

Mais, dans ces expériences, nous n'avions pas fait intervenir la chaleur en même temps que le formol, puisque nous avions opéré sur des laits formolés à la température ordinaire. 
Cette fois, nous avons pensé, afin de mieux juger l'action du formol sur l'emprésurage, à y associer l'influence de la température.

Quand on ajoute peu de formol au complexe, on ne nuit guère à sa coagulabilité par la présure, mais il n'en lest plus de même si la proportion du formol est grande et surtout si l'on chauffe.

Les portions égales d'un complexe $(p \mathrm{H}=6,78)$ formolé à raison de XXX gouttes par litre, sont chauffées 30 minutes respectivement à $60^{\circ}, 80^{\circ}, 100^{\circ}$ et à l'autoclave à $125^{\circ}$.

Après chauffage, on refroidit à $40^{\circ}$ et l'on emprésure.

TABLEAU XCIV.

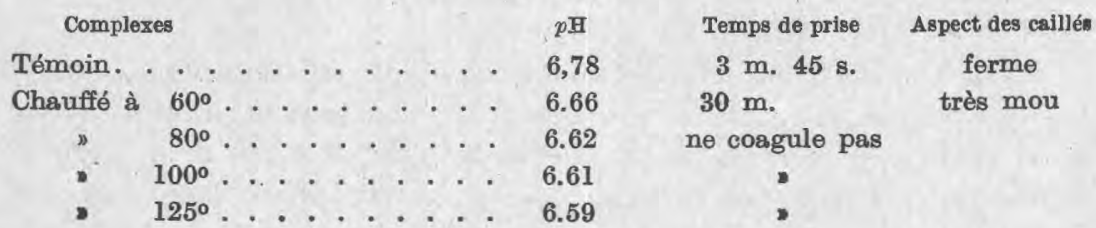

Toutefois, comme le chauffage à $100^{\circ}$ et a fortiori à $125^{\circ}$ n'a pas manqué d'altérer les phosphates calciques, il importe de séparer ce qui revient à ces derniers de ce qui dépend du caséinate. On y arrivera de deux façons[différentes :

a) En rechargeant en phosphates de calcium neufs le complexe formolé 1t chauffé;

b) En effectuant la charge phosphatique après chauffage d'un caséinate formolé voisin de la neutralité.

Voici que ce donne la première manière :

\section{TABLEAU XCV.}

Complexes

$p$ H

Témoin . . . . . . . . 6,78

Chauffé à $60^{\circ}$......... 6,78

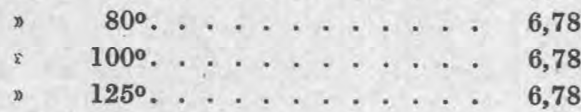

Temps de prise Aspect des calllé $6 \mathrm{~m}, 30 \mathrm{~s}$. ferme

$9 \mathrm{~m}, 15 \mathrm{~s}$. mou

$78 \quad 9$ mo

Voici ce que donne la seconde, avec un caséinate témoin $(p H=7,50)$ et toujours $\mathrm{XXX}$ gouttes de formol par litre de liqueur :

TABLEAU XCVI.

Nombre
de gouttes
de formol $p$ H des caséinates

\begin{tabular}{|c|c|c|c|}
\hline $\begin{array}{l}\text { Avant le } \\
\text { chauffage }\end{array}$ & $\begin{array}{l}\text { Après le } \\
\text { chauffage }\end{array}$ & $\underset{\text { complexe }}{p \text { H du }}$ & $\begin{array}{l}\text { Temps } \\
\text { de prise }\end{array}$ \\
\hline 7,50 & 7,50 & 6,83 & 60 s. \\
\hline 7,27 & 7,11 & 6,76 & $2 \mathrm{~m}$. \\
\hline 6,78 & 6,70 & 6,61 & ne coagule \\
\hline 6,69 & 6,61 & 6,62 & 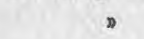 \\
\hline
\end{tabular}

6,62
Aspect des calllén

fermo

ferme

10 ......

30 
Cette dernière expérience témoigne indiscutablement d'une altération du caséinate, les phosphates n'ayant pas été exposés à être lésés.

\section{Mécanisme de l'insensibilisation progressive du complexe phosphatique à la présure sous l'action de la chaleur. - Cette} dissection des sensibilités propres au caséinate et aux phosphates de calcium ayant été faite, nous devons chercher à comprendre ce qui se passe exactement dans le complexe phosphatique lorsque, sous l'action d'une température assez élevée, il perd le pouvoir de coaguler par la présure. Nous n'avons pas à faire intervenir l'acidité, puisque nous voyons que l'action de la ehaleur a justement tendance à diminuer le $p H$, c'est-à-dire à l'enfoncer davantage dans la zone acide, tendance faible il est vrai, mais incontestable.

Nous avons vu dans la mécanique chimique des phosphates que si les phosphates tri- et mono-calcique sont relativement stables vis-vis de la chaleur, il n'en est pas de même du phosphate bi-calcique qui se décompose en phosphate tri-calcique et en phosphate mono-calcique dont la réaction acide est forte. Le phosphate mono-calcique libéré va, à son tour, réagir sur le caséinate calcique, s'emparer d'une partie de sa chaux, faire du phosphate tricalcique puisqu'on opère à une température élevée et conduire en même temps à l'obtention d'un caséinate calcique moins chargé en chaux que le caséinate originel, par conséquent légèrement plus acide.

En somme, quand nous voyons le phosphate monocalcique donner du phosphate tricalcique sous l'action de la chaleur aux dépens du caséinate calcique du complexe, nous pouvons dire que le caséinate calcique sert en quelque sorte ici de tampon.

Le glissement du $p H$ du complexe vers l'acidité sous l'action de la chaleur est donc le fait indirect de la libération première de phosphate monocalcique par décomposition du phosphate bi-calcique et le résultat direct de la présence dans la liqueur d'un caséinate plus acide que le caséinate de départ. D'ailleurs, on peut le prouver directement. Il suffit d'ajouter à un caséinate de $p \mathrm{H} \leqslant 7$, une solution de phosphate monocalcique, par apports successifs croissants. Cela nous permettra deux constatations :

a) La première: Apprécier à partir de quel moment la coagulation a lieu, car nous verrons, si la quantité de sel soluble n'est pas assez forte, que l'action première de la présure : le dédoublement du caséinate avec mise en liberté de protéose s'effectue bien, tandis que la précipitation du paracaséinate reste en suspens;

b) La seconde : Voir que la première association du caséinate et du phosphate monocalcique qui coagule nettement par la présure, ne coagulera plus lorsqu'elle est soumise à l'action de températures élevées: 115 à $120^{\circ}$.

- L'intervention du phosphate de calcium dans la coagulation du lait 
par la présure a été soupçonnée depuis longtemps déjà, mais elle a été l'objet de nombreuses interprétations. E. DuccAux, lorsqu'il parlait d'une façon très générale du rôle des sels minéraux dans l'action de la présure, les uns pour la faciliter, les autres pour l'entraver, appelait ipso facto l'attention des chercheurs sur la nécessité de suivre de très près les conséquences des apports salins dans le lait.

Nous trouvons chez les auteurs qui se sont occupés de cette question des hésitations, des contradictions, des justifications expérimentales insuffisantes ; c'est parce qu'ils sont généralement partis d'une conception qui nous apparaît inexacte dans la distribution des composants normaux du lait. Nous ajouterons qu'il n'est pas toujours fait mention par eux des variations de réaction engendrées par ces apports salins. C'est là une notion de première importance qui a été bien mise en valeur par l'utilisation systématique $d u p H$. Mais les résultats de toutes les recherches sont là et il reste à les interpréter à la faveur des renseignements que nous avons tirés des expériences qui précèdent.

Briot a pensé, après Duclaux, au rôle du phosphate de calcium, et il s'exprime sur lui en termes qu'il est intéressant de rappeler :

"Le lait est une solution de phosphate de chaux, comparable à certains points de vue, à celle de VAUDIN. Ce phosphate de chaux, par lui-même, est susceptible de se précipiter, de se coaguler. L'albuminoïde du lait, la caséine, est, elle aussi, susceptible de coagulation. Les deux phénomènes de coagulation ne sont pas indépendants l'un de l'autre, ils s'influencent réciproquement.

"La présure, en modifiant la caséine, change les conditions de solubilité dans le lait de la caséine et du phosphate, et l'on a un coagulum mixte de caséine et de phosphate de chaux. Il est difficile de dire quelle est, des deux substances, celle qui entraîne l'autre.

"Il est remarquable que toute autre cause qui modifie les conditions de précipitation du phosphate modifie profondément les conditions de coagulation du lait. Toute cause qui supprime le phosphate de chaux du lait avant la coagulation empêche cette coagulation d'avoir lieu. Ainsi agissent les dézalcifiants, oxalates et fluorures alcalins. Toute cause qui augmente la stabilité de la solution du phosphate de chaux retarde ou empêche la coagulation. Ainsi agissent les eitrates alcalins.

"La coagulation du lait par la présure serait done un phénomène double. D'abord une modification de la caséine, puis une coagulation à proprement parler, c'est-à-dire un phénomène physique. Ces deux stades du phénomène sont indépendants, le premier pouvant se faire sans être suivi du second.

"Du reste, la formation d'un précipité de phosphate de chaux gélatineux dans le lait peut entraîner la caséine, même lorsqu'elle n'a pas été modifiée par la présure. 
« Si, à du lait, on ajoute du|phosphate disodique, et à ce lait phosphaté du chlorure de calcium, on a formation d'un précipité de phosphate de chaux gélatineux qui entraîne la caséine en un caillot forméde très gros grumeaux, presque compact, et qui laisse exsuder un sérum clair. Séparément, le phosphate de soude et le chlorure de calcium sont sans action à la température de $40^{\circ}$ à laquelle on opère.

"Si on se rappelle qu'il y a dans le lait du phosphate de soude (1), peut-être aura-t-on là une explication de l'action du chlorure de calcium... On augmente la quantité de phosphate de chaux du lait; on augmente par suite sa coagulabilité. La quantité de phosphate de chaux peut même devenir suffisante pour précipiter à l'ébullition, et alors entraîner la caséine et donner une coagulation ".

Et Briot, quand il résume ses recherches sur la présure, précise encore davantage le rôle du phosphate de chaux :

Je conclurai, dit-il :

" $1^{0} \mathrm{Au}$ dédoublement du phénomène de la coagulation du lait par la présure en deux actions, l'une de nature inconnue portant sur la caséine, I'autre qui constitue à proprement parler la coagulation et qui est de nature purement physique ;

« $2^{\circ} \mathrm{A}$ la corrélation très étroite de la deuxième action, de la coagulation proprement dite, avec la précipitation du phosphate de chaux du lait. Le coagulum n'est typique que lorsqu'il $y$ a encore de la chaux dans le lait. Ce serait donc le précipité de phosphate de chaux qui donnerait au coagulum de caséine son caractère de compacité, de rétractilité... "

Nous ne pouvons nier que ce texte avait imprégné notre esprit au point que sous l'empire des théories colloïdales, notre première explication de l'emprésurage se rapprochait nettement de celle de Briot. Nous avions parlé du phosphate de calcium en sous-entendant qu'il ne s'agissait que de phosphate tricalcique et, conséquemment, nous n'avions pas cherché à nous rendre compte si vraiment c'était uniquement du tricalcique qui se formait. Nos recherches ultérieures sur la distribution des trois phosphates calciques : mono-, bi- et tri-, sur les rapports qu'ils ont entre eux et dont la variabilité, encore une fois, est sous la dépendance de la réaction du milieu et de la température, nous ont fait changer d'opinion. Nous ajouterons à cela nos expériences sur la dialyse du complexe dont la signification est si grande, puisqu'elle met hors de cause les phosphates insolubles auxquels nous attribuions au début le rôle principal et qu'elle fait jouer, au contraire, aux phos-' phates solubles que nous négligions, le rôle essentiel.

L'acide phosphorique, dans le complexe, se partage de façon à donner du phosphate tricalcique, insoluble et non dialysable, et des phosphates solubles, mono- et bi-, dialysables. C'est cette distinction entre les deux

(1) En róalité, c'est de phosphate de potassium qu'il s'agit. (Ch. P,). 
sortes de phosphates qui n'avait pas été suffisamment faite jusqu'ici dans l'interprétation du mécanisme de la coagulation par la présure du complexe artificiellement préparé et du lait.

Redisons-le, si 'dans nos premières recherches nous n'avons pas souligné cette différence, c'est que nous pensions que le phosphate qui se formait était du tricalcique insoluble, et c'est pourquoi, guidé par des considérations physiques et sous l'empire des théories colloidales, nous avions été amené à attacher aux micelles phosphatiques insolubles une importance que nos expériences ultérieures leur ont entièrement enlevée.

Le chauffage du complexe caséinate de calcium + phosphate monocalcique. - Ces développements nous amènent à cette conclusion que si un complexe phosphatique, chauffé à une température suffisante, perd[sa Icoagulabilité par la présure, c'est que les phosphates calciques solubles se sont transformés en phosphates insolubles. Pour le démontrer, nous avons selon le protocole expérimental exposé ci-dessus, effectué les recherches suivantes :

Nous partons d'un caséinate de $p \mathrm{H}=7,29$, et nous l'additionnons en proportions variables de phosphate monocalcique dont la solution mère contient $2,34 \mathrm{gr} . \%$ $(\mathrm{N} / 10)$ et a un $p \mathrm{H}=3,4$. Nous faisons différents complexe: :

10 Un premier qui renferme $1,17 \mathrm{gr}$. de phosphate monocalcique au litre; son $p \mathrm{H}=6,62$;

$2^{\circ}$ Un second renfermant $0,58 \mathrm{gr}$. de phosphate au litre; $p \mathrm{H}=6,79$;

$3^{\circ}$ Un troisième contenant $0,30 \mathrm{gr}$. de phosphate monocalcique au litre; $p \mathrm{H}=6,94$;

Alors que le premier et le second font prise rapidement sous l'action de la présure, le troisième ne précipite pas et devient seulement porcelanique.

Pour voir de plus près quelle serait la dose minimum suffisante pour obtenir une coagulation, nous préparons un quatrième complexe ayant $0,45 \mathrm{gr}$. de caséine au litre; $p$ H $=6,88$, et un cinquième renfermant 0,50 gr. de sel au litre ; $p H=6,85$.

Alors qu'avec la dose de 0,45 gr., l'emprésurage aboutit à une coagulation incomplète, laissant le sérum encore laiteux, avec $0,50 \mathrm{gr}$. nous obtenons un bloc net, ferme et un sérum limpide.

Si nous portons le cinquième complexe à l'autoclave à $115^{\circ}$ pendant 20 minutes, il ne coagule plus du tout par la présure et son $p \mathrm{H}=6,80$. Il a donc légèrement glissé vers l'acidité, Le phosphate mono-calcique s'est transformé en phosphate tricalcique aux dépens du caséinate qui, légèrement décalcifié, est devenu un peu plus acide.

On s'explique ainsi que l'incoagulabrlité acquise, tant par le complexe que par le lait, sous l'action de la chaleur, s'observe alors que ces deux liqueurs ont cependant viré vers l'acidité. La réaction semblerait donc favorable à l'emprésurage, mais par contre il n'y a plus assez d'ions Ca solubles. Une fois de plus s'établit la discrimination nécessaire dans les rôles respectifs des ions $H$ et des ions Ca au cours de l'emprésurage. 


\section{LES APPORTS SALINS DANS LE COMPLEXE PHOSPHATIQUE.}

Avant d'aborder l'étude d'autres complexes ou systèmes formés de l'union du caséinate avec des sels calciques, nous avons à examiner les modifications subies par le complexe phosphatique sous l'influence de divers apports salins. Nous avons résumé pareille étude dans des pages antérieures au sujet du lait: mais ici, toujours pour dégager le complexe de ce qui l'entoure dans le lait, nous l'isolons tout à fait et nous allons lui ajouter des sels calciques sous forme de chlorure ou des sels alcalins. Nous avons un peu plus haut rappelé que des modifications du $p H$ peuvent en résulter; nous aurons done à suivre les variations de la réaction du complexe sous l'influence des apports salins, puisque l'addition de tel sel entraîne le complexe vers l'acidité, de tel autre, au contraire, vers l'alcalinité.

ADDITION DE CaCI $^{2}$ AU COMPLEXE. - Cette addition fait virer le complexe vers l'acidité, légèrement mais d'une façon indéniable, en même temps qu'elle facilite l'emprésurage.

TABLEAU XCVII
a) Complexe .............. . . 7,05
id. $+\mathrm{CaCl}^{2}(0,80$ gr. par litre de complexe) $\quad 6,96$
b) Complexe ............ . . . 6, 6,80
id $+\mathrm{CaCl}^{2}$ (o,80 gr. par litre de complexe) $\quad 6,70$

Nous savons que l'addition de $\mathrm{CaCl}^{2}$ au simple caséinate blanchit celui-ci au point que si, auparavant, il était assez translucide il tend, après à ressembler au lait écrémé, surtout si on le porte à $40^{\circ}$. L'addition du même sel et dans les mêmes proportions au complexe phosphatique, déjà lui-même plus laiteux que le caséinate, provoque le même blanchiment et une tendance encore plus marquée à la floculation. En effet, nous augmentons la charge en calcium du milieu, ce qui tend à provoquer l'insolubilisation de sels déjà eux-mêmes peu solubles ou en suspension colloïdale.

La plus grande sensibilité du complexe au chlorure de calcium. - Lorsqu'on ajoute du $\mathrm{CaCl}^{2}$ au complexe phosphatique, on obtient un précipité, même à faible dose : 0,50 gr. par litre, mais il n'entraîne pas toute la caséine du complexe. Avec 0,50 gr., on précipite $20 \%$ de la caséine; avec 3,30 gr., $30 \%$; avec 5,50 gr., $70 \%$. Si l'on chauffe, la précipitation s'accentue et elle se complète en même temps que le sérum devient plus limpide.

Il est facile de comprendre qu'il faille moins de $\mathrm{CaCl}^{2}$ avec le complexe qu'avec le caséinate, puisque celui-là comporte déjà une certaine masse de phosphates calciques solubles, le mono- et surtout le bi-, qui viennent ajouter leur action à celle du chlorure. 


\section{LES SELS DECCALCIFIANTS.}

Nous avons vu que sous le nom de sels décalcifiants, on désigne les fluorures et les oxalates alcalins dont les sels calciques correspondants sont'insolubles. On sait que l'addition de ces sels au lait nuit à l'emprésurage, ainsi d'ailleurs que celle des citrates alcalins. Mais, on n'a pas toujours précisé le mécanisme du retard ou de l'absence de la coagulation. On a d'abord pensé que les fluorures ou les oxalates enlevaient la chaux qui était nécessaire à la précipitation du paracaséinate calcique; mais il faut aussi tenir compte d'une modification dans la réaction du milieu qui se traduit par une marehe telle vers l'alcalinité que celle-ci dépasse parfois par son $p H$ le point où l'action de la présure ne peut plus s'exercer.

ACTION DU FLuoruRe DE SODIUM. - A des quantités égales du même complexe, on ajoute des solutions de fluorure de sodium, de façon que les charges de ce sel soient respectivement par litre : 0,50 gr., 1 gr., 2 gr.

Voici les résultats :

\section{TABLEAU LXCVIII}

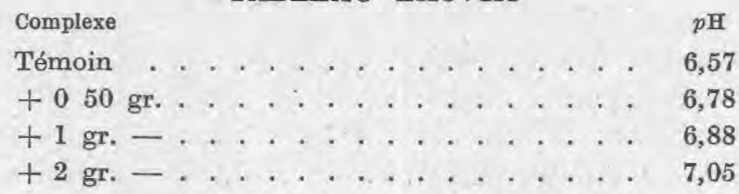

Comme toujours dans nos expériences, il est tenu compte des dilutions entraînées par l'addition de la solution de NaF.

Alors que le complexe témoin et les deux qui suivent ne présentent pas nettement de différences et ont conservé un aspect un peu laiteux, le complexe chargé avec 2 grammes de $\mathrm{NaF}$ par litre a perdu cette apparence laiteuse et est devenu opalescent, certainement parce que, du fait d'une double décomposition entre les sels calciques et le fluorure de sodium, il s'est fait du fluorure calcique, colloïdal sans doute, mais à côté, des caséinates et des phosphates alcalins.

ACTION DE L'OXALATE D'AMIMONIUM SUR LE COMPLEXE. - A un complexe de $\mathrm{pH}=6,88$, on a ajouté des quantités croissantes d'oxalate d'ammonium : 0,50 gr., 1 gr. et 2 gr. par litre.

Voici les résultats obtenus :

TABLEAU XCIX

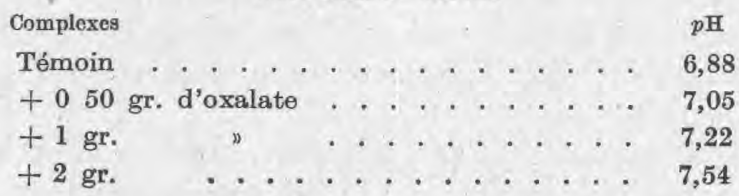

Le complexe chargé à raison de 2 gr. d'oxalate au litre est sensiblement plus blane que les autres; il a perdu son aspect opalescent. 
Alors que le témoin coagule par la présure, les complexes chargés en oxalate, même lorsque la charge n'est que de 0 ,gr. 50 , ne coagulent plus.

Il ne faut pas s'en étonner pour le complexe chargé à 2 gr. qui est nettement alcalin et dont la réaction est à la limite de celle qui est nécessaire à la présure pour agir.

La réaction du complexe additionné de $0,50 \mathrm{gr}$. et de $1 \mathrm{gr}$. d'oxalate traduite respectivement par des $p H=7,05$ et 7,22 , ne saurait empêcher l'action du lab, dans d'autres circonstances; mais ici, il y a une intervention non douteuse de l'oxalate lequel a précipité une partie du calcium soluble et également décalcifié une partie du caséinate qus a été transformé en caséinate alcalin.

Il s'agit donc ici, et d'une modification dans la réaction, et aussi, d'une action chimique entre l'oxalate et la chaux du complexe, que cette chaux appartienne aux phosphates ou au caséinate.

En effet, si nous ajoutons de l'acide oxalique au compiexe chargé de 2 gr. d'oxalate, nous ramenons bien son $p H$ à 6,90 , celui en somme du témoin, mais il ne coagule pas davantage. Pas davantage, également si au lieu d'ajouter de l'acide oxalique, nous prenons de l'acide phosphorique. Incontestablement, la chaux a été précipitée sous une forme insoluble.

L'expérience a été recommencée en mettant d'abord en présence les deux charges : 0 gr. 50 et 2 gr. d'oxalate au litre :

TABLEAU C.

$\begin{array}{ccc}\text { Complexe } & p \text { H } & \text { Temps de prise } \\ \text { Témoin } & 6,88 & 60 \text { sec } \\ +050 \text { gr. d'oxalate } & 7,05 & \text { Ne coagule pas } \\ +2 \text { gr. } & 7,55 & \text { Ne coagule pas }\end{array}$

Puis, cette fois, on revient au $p H$ initial avec HCl. Le complexe additionné de 0 gr. 50 d'oxalate fait prise par la présure en 5 minutes, mais le caillé est mou.

Si on revient au même $p$ H : 6,88 , non plus par addition d'HC1, mais par addition de $\mathrm{CaCl}^{2}$, le temps de prise est réduit à 60 secondes, et le caillé est ferme ; en même temps, le sérum est limpide, tandis que tout à l'heure avec $\mathrm{HCl}$, alors que le caillé était mou, le sérum était laiteux.

1 L'addition de $\mathrm{CaCl}^{2}$ a une tout autre signification que celle de $\mathrm{HCl}$. Celui-ci, bien qu'acide fort, ne solubilise à nouveau qu'une faible partie de la chaux précipitée, tandis que le $\mathrm{CaCl}^{2}$ apporte sous une forme soluble et en abondance de nouveaux ions $\mathrm{Ca}$ qui se sont substitués aux ions antérieurement solubles et que l'addition d'oxalate avait entraînés en les insolubilisant.

Si nous nous adressons au complexe chargé de 2 grammes d'oxalate, nous n'allons pas obtenir tout à fait les mêmes résultats, parce que l'apport de ce sel alcalin est trop considérable. L'addition de $\mathrm{HCl}$ n'amènera aucune coagulation par la présure ; celle de: $\mathrm{CaCl}^{2}$ provoquera 
à un moment donné, sans présure, une précipitation mixte d'oxalate de calcium et du complexe.

4. Si au lieu d'utiliser les oxalates alcalins qui apportent dans le com. plexe une alcalinité faisant entrer le $p H$ dans la zone alcaline, nous prenons l'acide oxalique qui apporte des ions $H$, nous allons voir que si de petites doses de cet acide facilitent la coagulation par la présure, de plus fortes doses l'entravent, malgré l'acidité, parce que justement il y a précipitation des sels calciques.

Nous dissocions une fois de plus, par cette simple expérience, les rôles respectifs des ions $\mathrm{Ca}$ et des ions $\mathrm{H}$.

ACTION DE L'ACide OXALiQue sur Le complexe, - Le complexe préparé comme à l'ordinaire est. additionné de $0,50 \mathrm{gr}$., puis de 1 gr. d'acide oxalique pour un litre et on emprésure.

TABLEAU CI.

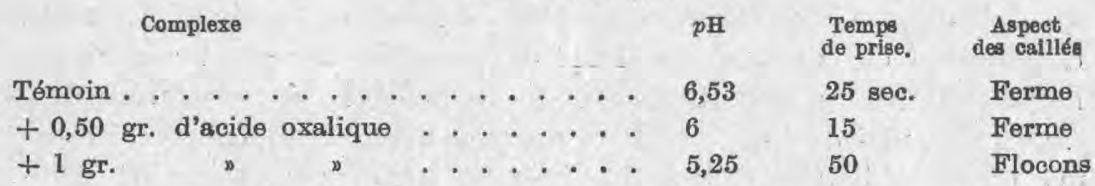

Avec une addition de 1 gramme, nous n'avons pas la prise en masse que nous avons obtenue avec le témoin et après addition de 0 gr. 50 d'acide oxalique. C'est qu'il y a, à froid, un commencement de précipitation d'oxalate de calcium ainsi que de caséine ou plus exactement d'un caséinate peu calcique, puisque le $p H$ est bas : 5,25. La présure amène la précipitation de petits coagulats qui n'ont pas tendance à se souder, et le sérum est toujours blanc laiteux.

AdDition du citrate dE potassium. - La charge du complexe en citrate de potassium aux mêmes doses que pour le fluorure de sodium et l'oxalate d'ammonium, le citrate étant calculé anhydre, nous donne des résultats analogues :

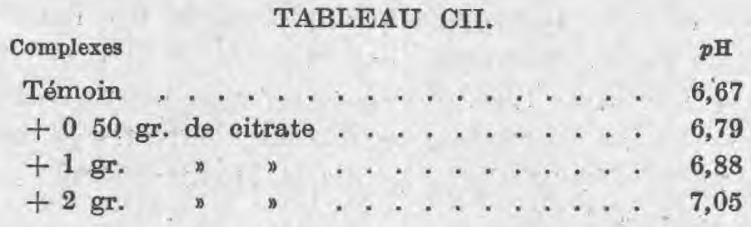

Il y a peu de différences à relever entre le complexe témoin et les deux complexes chargés de 0 gr. 50 et'de 1 gr. de citrate.

Le complexe à 2 gr. paraît un peu plus blanc; il ne faut pas en être surpris parce qu'il s'agit là d'une dose de citrate assez forte laquelle en réagissant avec les sels de calcium aboutit à une charge en citrate calcique telle qu'on est à la limite de la saturation de ce dernier sel qui, alors, va précipiter ; aussi les citrates alcalins 
jouent-ils le même rôle décalcifiant que les oxalates et les fluorures de la même famille.

Certains auteurs ont utilisé, avec le lait, des doses beaucoup plus considérables que celle de 2 gr. que nous avons employée avec le complexe, si bien qu'au point de vue moléculaire, l'importance des apports salins est telle qu'il y a des déplacements d'équilibre considérables.

A. W. Bosworth et L. L. VAN SLYKe (283) ont recherché pourquoi l'addition du citrate de sodium empêchait la coagulation du lait par la présure. Ils ont trouvé que ce sel solubilise la chaux, le phosphore et la magnésie et fait passer graduellement une partie du caséinate de calcium à l'état de caséinate de sodium; il est bon de noter que les quantités de citrate de sodium employées par eux étaient élevées. Partant de 1,30 gr. par litre de lait, elles s'élèvent progressivement à 10,40 gr. ; BRIOT allait jusqu'à 16 grammes.

Revenons sur une réflexion relative au même sujet afin d'en marquer une fois de plus l'intérêt : Quand, à un litre de lait, on ajoute 1 gramme de chlorure de calcium, soit $1 / 111$ de molécule de ce sel considéré à l'état anhydre, on apporte sous la forme soluble, 50 centigrammes de chaux, soit plus du $1 / 4$, en chiffres ronds de la chaux totale préexistante, que nous considérions celle-ci sous la forme soluble ou la forme insoluble. N'est-ce pas là une proportion importante qui nous permet de préjuger du rôle qu'elle peut jouer? Aussi, quand on ajoute les quantités élevées de citrate de sodium dont parlent Bosworth et VAN SLYKE, puis BRIOT, on déplace l'équilibre salin én faveur des sels sodiques, d'une façon telle qu'il n'est pas surprenant que le lait ne réagisse plus à la présure.

Si nous avons, en passant, parlé du citrate de sodium, e'est parce que justement, dans la diététique infantile, on ajoute ce sel au lait de vache pour le rendre difficilement coagulable par la présure et le rapprocher du lait de femme, de ce côté du moins.

Ainsi qu'il résulte des expériences ci-dessus, on voit que, en dépit, de leur forme colloïdale, le caséinate de calcium et les phosphates caleiques insolubles du complexe, et par extension disons du lait, réagissent avec ces sels de telle façon que le déplacement des équilibres salins est régi par les lois de GULDBERG et WAAGE.

Cette observation est d'ordre général et nous pouvons la renouveler avec des sels alcalins autres que les fluorures, citrates et oxalates, sels qui n'amènent aucune précipitation de la chaux, les sels calciques de leurs anions étant solubles.

LE ROLE dU SEL (NaCI) DES PRÉSuRES Liquides. - Lorsque nous utilisons la présure du commerce non dialysée laquelle est extrêmement riche, comme on le sait, en chlorure de sodium, puisque le taux de ce

(283) A W. Bosworth et L.L. VAN SLYKe, - Why sodium citrate prevents Curdling of Milk by Rennin ? N. Y. Agr. Exp. Stat. 1914. - Techn. Bull. no 34 et The Amer, J. of Dis. of Children, 1914, 7, 298. 
sel y atteint $25 \%$, nous constatons que l'addition de cette présure à la dose habituelle à un caséinate acide et légèrement laiteux, le rend tout à fait translucide. C'est qu'il y a eu double décomposition entre le caséinate de calcium et $\mathrm{NaCl}$ et formation d'un nouveau système en équilibre renfermant caséinate de sodium, caséinate de calcium, $\mathrm{NaCl}$ et $\mathrm{CaCl}^{2}$.

Toute charge saline d'un métal alcalin $\left(\mathrm{K}, \mathrm{Na}, \mathrm{NH}^{4}\right)$ aboutit au même résultat lorsque, par double décomposition entre le caséinate de calcium et le sel, il y a tendance à la formation d'un sel calcique soluble.

Iodure, nitrate, nitrite et chlorure de potassium rendent immédiatement le caséinate auquel on les ajoute tout à fait translucide. Il en est de même à froid, lorsqu'il s'agit d'un phosphate, d'un carbonate ou d'un sulfate. Mais quand on chauffe, nous notons des différences.

A $40^{\circ}$, les tubes additionnés d'iodure, de nitrate, de nitrite et de chlorure se modifient peu; au contraire, ceux additionnés de phosphate, de carbonate et de sulfate se troublent et deviennent un peu blancs, parce qu'il y a formation de sels correspondants de calcium insolubles.

Cette simple expérience est pleine d'enseignements, car elle nous montre que la chaleur même modérée entraîne facilement des déplacements d'équilibre salins vers l'insolubilisation des sels calciques.

Nous jugeons ainsi tout ce que nous pouvons en tirer relativement à l'étude de la mécanique chimique du lait. Les lois n'en sont nullement modifiées par l'état colloïdal des sels qui peuvent jouer les uns sur les autres; encore une fois, l'allure de la réaction n'est pas altérée, c'est simplement son aspect qui peut être modifié.

L'action du $\mathbf{N a C l}$ sur les caséinates. - Il est intéressant de se limiter au sel le plus courant, c'est-à-dire $\mathrm{NaCl}$, et d'étudier son action sur le caséinate.

A $20 \mathrm{~cm}^{2}$ de caséinate à $500(1,66 \%$ de chaux $)$, nous avons ajouté $0,055 \mathrm{gr}$. de $\mathrm{NaCl}$ et mis à dialyser contre de l'eau fixe. Un témoin est placé dans les mêmes, conditions, mais en l'absence de $\mathrm{NaCl}$. On porte à la glacière.

Après 18 heures de dialyse, le caséinate témoin a gardé gon aspect blane laiteux mais le caséinate chloruré est devenu plus limpide; il est très opalescent.

On dose la chaux dans l'eau des dialyseurs. Le caséinate témoin n'en a pas laissé passer la moindre trace, alors que le caséinate chloruré a laissé passer, pour $20 \mathrm{~cm}^{3}$ de caséinate dialysé, $0,0096 \mathrm{gr}$. de chaux.

Or, la chaux correspondant aux $20 \mathrm{~cm}^{3}$ du caséinate était de $0,010 \mathrm{gr}$.

Puisque presque toute la chaux a dialysé, c'est qu'une double décomposition est intervenue entre le caséinate de calcium et le chlorure de sodium. Celle-ci a été presque totale, parce que, en face de $0,010 \mathrm{gr}$. de chaux, nous avions $0,055 \mathrm{gr}$. de $\mathrm{NaCl}$, e'est-à-dire, au point de vue moléculaire, 1/6 d'une molécule de chaux, en chiffres ronds, s'opposant à presque une molécule de chlorure de sodium. 
Il importait de doser parallèlement le chlore dans l'eau du dialyseur. On en a retrouvé, traduit en $\mathrm{NaCl}, 0,0505 \mathrm{gr}$. au lieu de $0.055 \mathrm{gr}$., soit presque tout le chlore apporté par $\mathrm{NaCl}$.

Si la dialyse s'était effectuée contre de l'eau courante, nous n'aurions plus retrouvé dans le dialyseur que du caséinate de sodium.

L'emploi du $\mathbf{C a C l}^{\prime}$ dans I'industrie laitière. - A l'occasion des doubles décompositions ci-dessus, il est bon d'entrer dans quelques considérations qui, une fois de plus, vont nous faire comprendre que ee sont les sels solubles de calcium qui jouent le rôle nécessaire dans la gélification labique.

L'emprésurage est amélioré, nous le savons, tant du côté de sa rapidité que du côté de la consistance du caillé, par l'addition de $\mathrm{CaCl}^{2}$. Le fait est connu depuis longtemps. E. Duclaux, BRtot... qui, systématiquement, ont étudié l'influence des sels les plus variés des séries al'caline, alcalino-terreuse et magnésienne sur l'emprésurage, ont noté l'action hautement favorisante du $\mathrm{CaCl}^{2}$. L. LINDET $(a)($ 284, 285) dès 1913, constate, de plus, au point de vue industriel, que le rendement, du caillé en matière azotée et en acide phosphorique est augmenté.

La pratique d'ajouter du $\mathrm{CaCl}^{2}$ au lait pour en faciliter l'emprésurage est aujourd'hui assez 'courante en fromagerie (VAmLANT (286,), CAMPS (287).

Le chlorure de calcium augmentant l'acidité du milieu fait baisser le $p H$, relativement peu lorsqu'il s'agit du complexe, davantage lorsqu'il s'agit du lait. Déjà, la seule accentuation de la réaction acide est favorable à l'action de la présure, mais il ne faut pas oublier, lorsqu'on s'adresse au lait, qu'il existe dans cette sécrétion des phosphates alcalins, notamment $\mathrm{du}$ phosphate bipotassique, lequel réagira avec $\mathrm{CaCl}^{2}$ pour donner du phosphate bicalcique qui viendra s'ajouter au même sel originel. Quand on apporte $d u C a C l^{2} \grave{a}$ un lait qu'on emprésure, on retrouve dans le sérum moins d'acide phosphorique que dans le sérum du lait témoin. Il y a également une perte en calcium, c'est-à-dire que la chaux trouvée est inférieure à la somme de la chaux du sérum du lait témoin et de la chaux introduite avec le $\mathrm{CaCl}^{2}$.

(a) Aux publications déjà signalées de L. LINDEx ajouter :

(284) L. LINDEx. - Sur la coagulation de la caséine en présence des selșde chaux en solution acide. - Le Lait, 1925, 5, 953.

(285) L. Lindex. - Solubilité des albuminoídes du lait dans les éléments du sérum; rétrogradation de la solubilité sous l'influence du chlorure de calcium. - Rev. Gén, du Lait. 1911-1914, 9, 373.

(286) E. Varllañt. - L'emploi du chlorure de calcium dans la fabrication du fromage de Maroilles. - Le Lait, 1924, 4, 7.

(287) Canrs. - L'emploi du chlorure de calcium dans la fromagerie au Danemark. Le Lait, 1924, 4, 895. 
Le tableau ci-dessous donne les chiffres des expériences :

TABLEAU CIII.

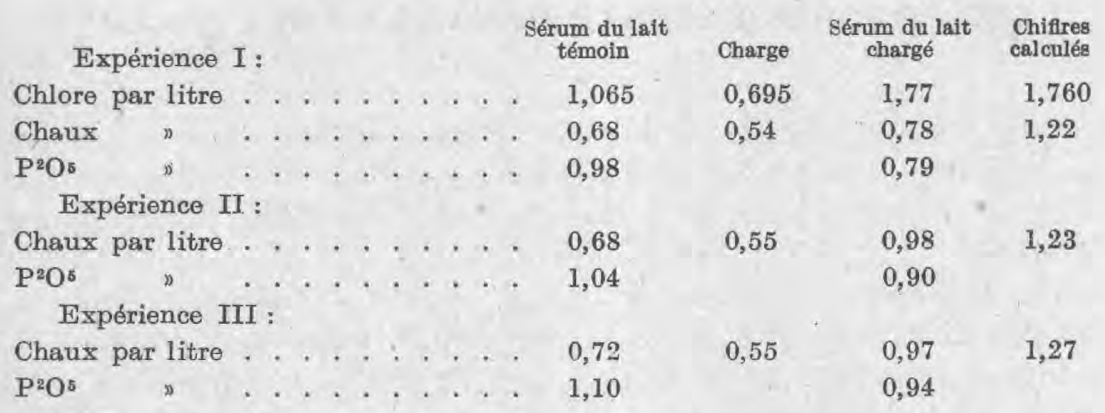

On voit que l'on retrouve dans le sérum tout le chlore ajouté sous la forme de $\mathrm{CaCl}^{2}$, ce qui ne saurait nous surprendre, mais il y a une perte en $\mathrm{CaO}$ et en $\mathrm{P}^{2} \mathrm{O}^{5}$ proportionnellement plus importante pour la base que pourl'acide. Dans l'hypothèse où il se ferait du phosphate bi-caleique, la perte en $\mathrm{P}^{2} \mathrm{O}^{5}$ serait supérieure à celle en $\mathrm{CaO}$ (142 contre 112); s'il s'agissait de phosphate tricalcique, elle serait un peu inférieure (142 contre 168); or, dans les trois expériences, la perte en $\mathrm{P}^{2} \mathrm{O}^{5}$ est, respectivement, les $43 / 100$, les $56 / 100$ et les $52 / 100$ de la perte en chaux. On est autorisé à conclure que la chaux qui disparaît n'emprunte pas uniquement la forme phosphatée; le chlorure de calcium ajouté doit done réagir avec d'autres sels que les phosphates alcalins. Il y a dans le lait des citrates alcalins qui avec $\mathrm{CaCl}^{2}$ donnent du citrate de Ca lequel s'ajoute aux citrates originels ; mais comme le taux du citrate calcique préformé est voisin de la saturation, tout citrate calcique surajouté doit précipiter. Quoi qu'il en soit, l'addition de $\mathrm{CaCl}^{2}$ au lait n'entraîne pas une totale précipitation de la chaux soluble qu'on lui apporte; la précipitation n'est que partielle. Il restera donc un résidu soluble de chaux qui interviendra pour faciliter l'emprésurage dans sa seconde phase : la précipitation du paracaséinate.

L. LTNDET admettait la formation nouvelle, par addition de $\mathrm{CaCl}^{2}$ au lait, de phosphate de calcium et de citrate de calcium. Il les retrouve, en effet, dans le caillé d'emprésurage dont ils augmentent la charge minérale par rapport à celle du caillé témoin; mais, encore une fois, toute la chaux nouvelle apportée sous forme de $\mathrm{CaCl}^{2}$ ne se retrouve pas dans les précipités calciques nouveaux; elle s'est partagée inégalement entre eux et le sérum. C'est sa forme soluble, et non sa forme insoluble, qui accélère l'emprésurage en le rendant plus complet.

(A suivre). 